\title{
MiR-29b Inhibits Ventricular Remodeling By Activating Notch Signaling Pathway in the Rat Myocardial Infarction Model
}

\author{
Yang Liu, ${ }^{1}$ Hongliang Wang, ${ }^{2}$ Xiudan Wang, ${ }^{3}$ Guohong Xie ${ }^{4}$ \\ ${ }^{1}$ Department of Child Healthcare, Linyi People's Hospital, NO. 233, Fenghuang Avenue, Jiuqu Community, Hedong District, Linyi \\ City, 276000, Shandong Province, China; ${ }^{2}$ Department of Cardiology, Jinan First People's Hospital, NO. 132, Daming Lake Road, Lixia \\ District, Jinan City, 250000, Shandong Province, China; ${ }^{3}$ Department of Pneumology, Jinan Third People's Hospital, NO. 1, North \\ Street, North Industrial Road, Licheng District, Jinan City, 250101, Shandong Province, China; ${ }^{4}$ Department of Cardiology, Jinan Central \\ Hospital Affiliated to Shandong University, NO. 105, Jiefang Road, Lixia District, Jinan City, 250013, Shandong Province, China
}

\section{ABSTRACT}

Background: To study the effect of miR-29b on myocardial infarction via Notch signaling pathway in rats.

Methods: The rat acute myocardial infarction (AMI) models were established and were divided into AMI group, sham group and normal group ( $N=10$ in each group). HE (Hemotoxylin and eosin) staining was used to detect whether the model was constructed successfully. MiR-29b mimics, inhibitors, mimics negative control (NC) were transfected into H9c2 (2-1) cells. Then, cells were divided into a mimics group, inhibitor group, NC group, and blank group. The relative expression levels of miR-29b, Notch1, DII4 and Hesl were detected by qRT-PCR. The expression of NICD1 was detected by Western blotting.

Results: The rat AMI model was successfully constructed. Compared with normal and sham groups, the miR$29 \mathrm{~b}$ expression was down-regulated, while the expression of Notch1, DII4 and Hesl was increased, and the NICD1 protein expression was increased in the myocardial infarction area of the AMI group $(P<.05)$. Compared with the NC and blank groups, the relative expression of Notch1, DII4, Hesl and NICD1 were upregulated in the mimics group $(P<.05)$, whereas the expression of Notch1, DII4, Hesl and NICD1 in the inhibitor group was decreased $(P<.05)$.

Conclusion: MiR-29b inhibited myocardial fibrosis and cardiac hypertrophy by activating the Notch signaling pathway and protected myocardium against myocardial infarction.

\section{INTRODUCTION}

Acute myocardial infarction (AMI), caused by coronary artery occlusion, may lead to myocardial ischemia and necrosis [Yanbing 2016]. Ventricular remodeling after AMI is attributed

Received fune 28, 2018; received in revised form November 15, 2018; accepted November 15, 2018.

Correspondence: Guohong Xie, Department of Cardiology, Finan Central Hospital Affiliated to Shandong University, NO. 105, Fiefang Road, Finan City, 250013, Shandong Province, China; +86-0539-8081739; (e-mail: xieguohong256@163.com). to the activation of the neuroendocrine system by necrotic myocardium, resulting in the structural and functional remodeling of cardiac myocytes, myocardial fibroblasts, and extracellular matrix [Zhang 2010]. Inhibiting ventricular remodeling after AMI improves the survival and quality of life, but its mechanism is not yet fully elucidated [Wang 2016].

In previous study, the Notch signaling pathway was found to play a vital part in the occurrence, development, physiology and pathology of cardiovascular system [Gopalakrishnan 2014]. The Notch signaling pathway is composed of three parts: Notch receptor (Notch1), Notch ligand (Jagged1, DII 4), and nuclear effector (Hesl). When the ligand is combined with the extracellular domain of Notch, the soluble Notch intracellular NICD is released. Recent studies have shown that Notch1 played an important role in the process of ventricular remodeling and participated in the process of cardiac fibrosis [Li 2011]. For instance, Nistri et al confirmed that Notch signaling had been a suitable target for inhibiting fibrosis in heart [Nistri 2017]. In addition, $W u$ et al found that Jagged 1 could protect against myocardial infarction by activating Notch signaling [Wu 2017]. Furthermore, Relaxin could prevent cardiac fibrosis by activating Notch signaling [Zhou 2015].

MicroRNA (miR) family has been confirmed to regulate the expression and translation of many genes in positive or negative direction, and play a vital part in the process of cell differentiation, apoptosis, tumor, growth and development [Smrčka 2016. Importantly, researchers also found the role of miRNA in fibrotic diseases. Two recent studies have confirmed that miR-26a/b and miR-30d could inhibit fibrosis by regulating Notch signaling pathway [Chen 2017; Zhao 2018]. Interestingly, miR-29b has been proven to be part of in the process of myocardial fibrosis after myocardial infarction [Yang 2015]. However, the mechanism of miR-29b and its related signaling pathways were unclear.

In this study, the regulation of miR-129 and Notch signaling pathway in ventricular reconstruction after infarction were initially studied. To understand the mechanism of mir-29b in ventricular reconstruction after infarction, we studied the miR$29 \mathrm{~b}$ expression in the myocardial tissue of a myocardial infarction rat model. The animal model was mainly used to detect the expression of MiR-29b and the Notch signaling pathway related genes in vivo. In the meantime, we also studied the effect of miR-29b on the Notch signaling pathway in myocardial cell 


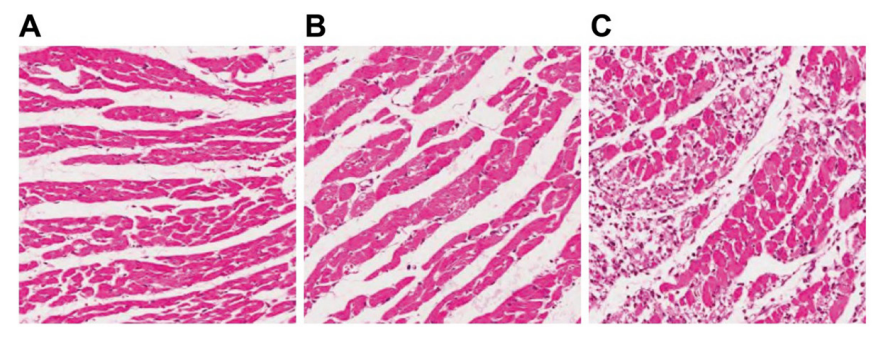

Figure 1. HE-staining of rat myocardial tissue. (A): Sham group; (B): Normal group; (C): AMI group. $N=5$ in each group.

line H9c2 (2-1). The experiments in vitro were processed to confirm the expression of above factors and the regulated relationships. Vivo and vitro experiments were combined to verify the effect of miR-29b on myocardial infarction.

\section{MATERIALS AND METHODS}

Animals and grouping: A total of 30 SPF adult male rats (14 weeks to 16 weeks; $180 \mathrm{~g}$ to $220 \mathrm{~g}$ in body weight) were provided by the experimental animal center of Peking University. The rats were randomly separated into an AMI group, sham group, and normal group ( $N=10$ in each group). The rats in the normal group did not perform any operation. Half of the rats' hearts in each group were freshly taken for qRT-PCR and Western blotting, and the other half were fixed for Hemotoxylin and eosin (HE) staining.

All animal experiments were in compliance with national animal experimental standards.

Construction of acute myocardial infarction model and grouping: The rats were anesthetized and laid on an experimental table in the supine position. Then, tracheotomy and tracheal intubation were performed, and a ventilator was used to assist respiration with a tidal volume between $6 \sim 8 \mathrm{~mL} / \mathrm{kg}$. The rat's chest was opened at the bifid third rib on the left side of the sternum, then the heart was exposed. The rats in the sham group were directly sutured. The 5-0 suture was used to ligate the left anterior descending coronary artery located $2 \mathrm{~mm}$ below the pulmonary artery cone and left atrial appendage junction in the rats of the AMI group. The hearts were taken out after 24 hours.

HE staining: The myocardium was fixed in $10 \%$ polyoxymethylene for 24 hours, dehydrated, permeabilized, and encased in paraffin. Before dying, paraffin sections $(5-6 \mu \mathrm{m})$ were dewaxed in xylene for $10 \mathrm{~min}$, and then dehydrated in high-concentration to low-concentration alcohol. These sections were dyed with hematoxylin for three min, and the color was separated in acid and ammonia water for 10 seconds respectively, then washed in stream for one hour, and respectively dehydrated in $70 \%$ and $90 \%$ alcohol for $10 \mathrm{~min}$. Then, these sections were dyed with eosin for three min, dehydrated, permeabilized, and sealed by neutral balata. The morphology changes of myocardium were observed under the microscope.

Cell line and culture: H9c2 (2-1) cell line was bought from the Shanghai cell bank (Shanghai, China), cultured in Dulbecco's modifed Eagle's medium (DMEM, Thermo Fisher Scientific, USA) with $10 \%$ fetal bovine serum (FBS), and incubated in a humidified chamber at $37^{\circ} \mathrm{C}$ with $5 \% \mathrm{CO}_{2}$.
Primer sequence for RT-PCR

Gene Primer sequence

miR-29b

F: 5'-ACACTCCAGCTGGGTAGCACCATTTGAAA-3'

R: 5'- TGGTGTCGTGGAGTCG-3'

U6

F: 5'-CTCGCTTCGGCAGCACA-3'

R: 5'-AACGCTTCACGAATTTGCGT-3'

NC

5'-CAGUACUUUUGUGIAGUAGUACAA-3'

Notch1

F: 5'-TCGCCGCAAGAGGCTTGAGATGCT-3'

R: 5'-TCGCTGCAGCACAGGCTTCA-3'

DII4

F: 5'-ATGCTCTTGCCGACCCGGGTAT-3'

R: 5'-TGCACACGTCATGGCGCTCA-3'

Hesl

F: 5'-CTGCCAAGGTTTTTGGCGGCT-3'

R: 5'-ACACTTGCGTTAGGACCCACCGA -3'

$\beta$-actin

\section{F: 5'-CCACGAAATACCTTCAACTCC-3'}

R: 5'-ACTCGTCATACTCCTGCTTGCT -3'

MiR-29b mimics, inhibitors and mimics negative control transfection: MiR-29b mimics, inhibitors, and mimics negative control (NC) were synthesized by Beijing Genomics Institute (Shenzhen, China). The primer sequences were as followed: miR-29b mimics $\mathrm{F}$ : 5'-ACACTCCAGCTGGGTAGC-ACCATTTGAAA-3'; R: 5'-TGGTGTCGTGGAGTCG-3'. miR-29b inhibitor 5'-AACACUGAUUCAAAUGGUGCUA-3'. NC 5'-CAGUACUUUUGUGUAGUAG-UACAA-3'. H9C2 (2-1) cells were cultured in 6-well plates to $70 \%$ confluence. According to the instructions of Lipofectamine 2000 (Invitrogen), miR-29b mimics, inhibitor and mimics negative control were added to the cell culture medium separately. Then the cells were cultured at $37{ }^{\circ} \mathrm{C}$ incubator with $1 \% \mathrm{O}_{2}, 94 \% \mathrm{~N}_{2}$, $5 \% \mathrm{CO}$, for 24 hours. The cells of the blank group were incubated at $37^{\circ} \mathrm{C}$ in a humidified chamber with $5 \% \mathrm{CO}_{2}$.

qRT-PCR: Total RNA of myocardium in each group was isolated by GenElute Mammalian Total RNA Miniprep Kit (Seebio, Shanghai, China). The cDNA template was reversetranscribed by the PrimeScript RT reagent kit (Takara, Tokyo, Japan). qRT-PCR was processed using the TaqMan miRNA detection kit. All procedures were performed following the manufacturer's instructions. U6 snRNA was used as reference for miRNA-29b, and $\beta$-actin was used as reference for Notch1, DII and Hesl. The method of $2 \wedge(-$ Delta Delta CT) was used to analyze the relative expression of gene. The primers for qPCR are shown in the Table. 
A

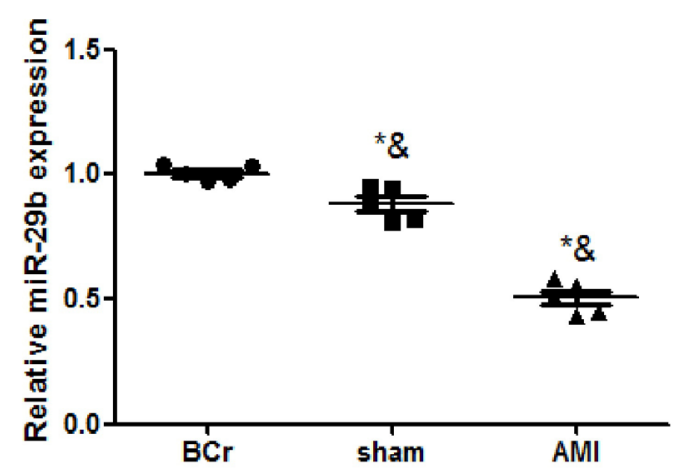

B

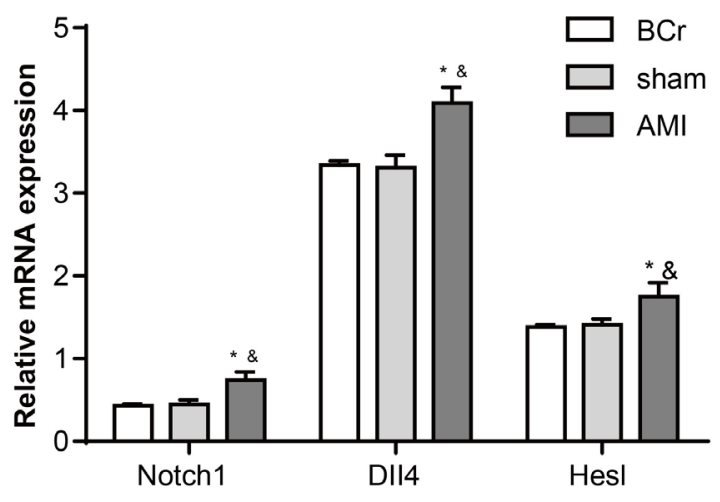

Figure 2. The expression of miR-29b, Notch1, Dll4 and Hesl in rat myocardium tissue. (A): The miR-29b expression; (B): The expression of Notch1, DII4 and Hesl. $\mathrm{N}=5$ in each group; Compared with normal group, ${ }^{*} P<.05$; Compared with sham group, $P<.05$.

A

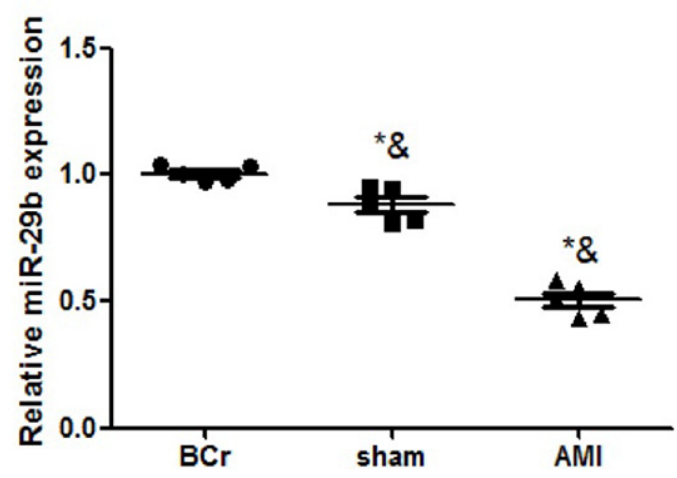

B

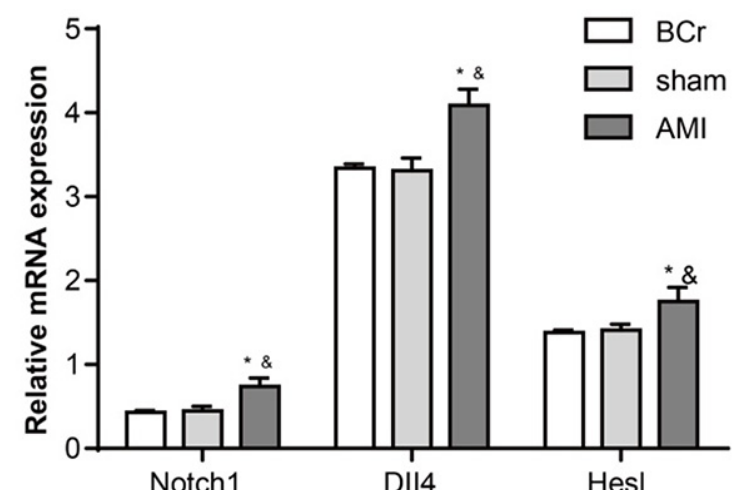

Figure 3. The expression of miR-29b, Notch1, DII4 and Hesl in H9C2 (2-1) cells. (A): The miR-29b expression in H9c2 (2-1) cells; (B): The expression levels of Notch1, Dll4 and Hesl in H9c2 (2-1) cells. $\mathrm{N}=5$ in each group; Compared with blank group, *P<.05; Compared with NC group, $P<.05$.

Western blot: Total protein was extracted from myocardium and H9c2 (2-1) cells, then detected by BCA Protein Assay Kit (Pierce, Shanghai, China). Subsequently, the protein was subjected to SDS-PAGE, and transferred onto the membranes. The NICD1 antibody (1:1000, ab52301 Abcam Britain) and horseradish peroxidase-labeled secondary antibody (1:5000, Beijing Zhong Shan Biotechnology Co., Ltd., China) were then added followed by incubation. $\beta$-actin was used as an internal reference.

Statistical analysis: All data were represented by mean values \pm standard deviation, and processed by SPSS 21.0 (SPSS Inc, Chicago, IL, USA). The comparison of data between groups was used Student-Newman-Keul's test. $P$ value less than 0.05 was the threshold of significant difference.

\section{RESULTS}

Establishment of the rat acute myocardial infarction mode: The myocardial cells $(40 * 20)$ were arranged in a regular pattern with the same size, the nucleus of myocardial cells were observed clearly, and cell swelling was not observed in the sham or normal groups. In the AMI group, many myocardial cells swelled and displayed necrosis, scattered in the necrotic foci with myosin degeneration and myocardial dissolution. It suggested the rat acute myocardial infarction mode was successfully established (Figure 1).

Expression of miR-29b, Notch1, DII4 and Hesl in myocardial infarction: After 24 hours of modeling, there was no significant difference in the expression levels of miR29b, Notch1, DII4 and Hesl between the sham and normal groups. The relative miR-29b expression was decreased in the AMI group $(0.51 \pm 0.05, P<.05$ versus the sham and normal groups). It indicated that miR-29b might play a role in rats with myocardial infarction (Figure 2A). Meanwhile, the expression levels of Notch1 $(0.82 \pm 0.06)$, DII4 (4.03 \pm 0.07$)$, and Hesl $(1.75 \pm 0.06) \mathrm{mRNA}$ in the AMI group were upregulated significantly $(P<.05$ versus the sham and normal groups). Thus, the Notch signaling pathway was activated in rats with myocardial infarction (Figure 2B).

Expression of miR-29b, Notch1, DII4 and Hesl in H9c2 (2-1) cells: After transfected for 24 hours, there was no 

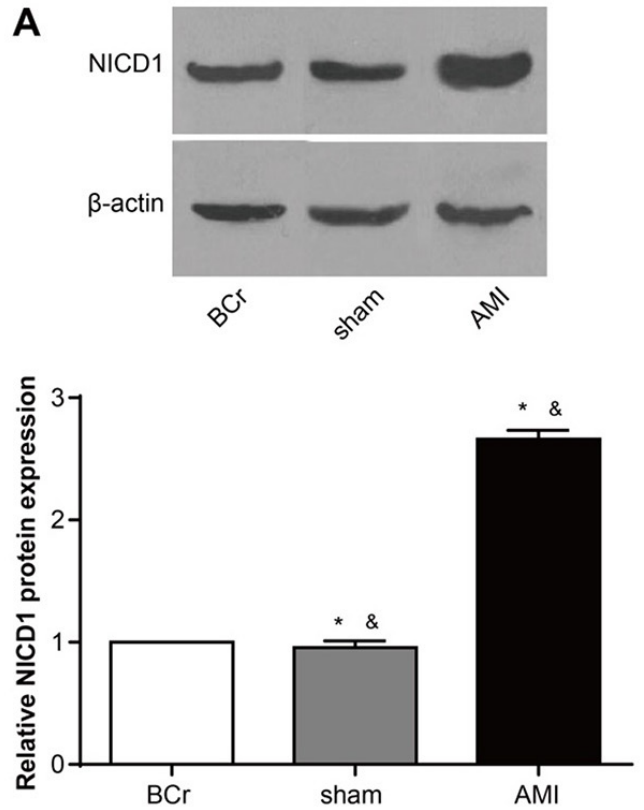
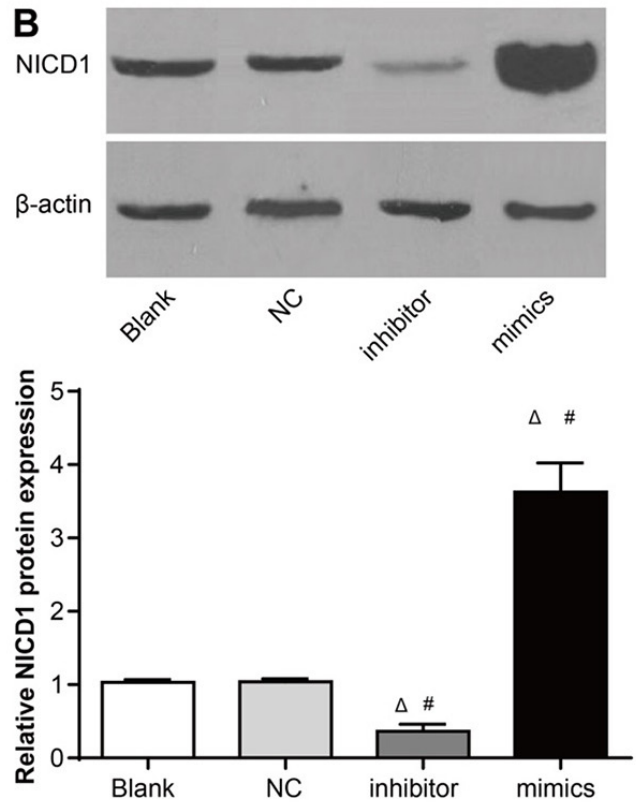

Figure 4. The expression of NICD1 protein (A): The NICD1 expression in rat myocardium tissue was detected by western blotting; (B): The NICD1 expression in $\mathrm{H} 9 \mathrm{c} 2$ (2-1) cells was detected by western blotting; $\mathrm{N}=5$ in each group; Compared with normal group, * $P<.05 ;$ Compared with sham group, $P<$ 0.05; Compared with blank group, \#P<.05; Compared with NC group, $\Delta P<.05$.

significant difference in the expression levels of miR-29b, Notch1, DII4 and Hesl between the NC group and blank group. Compared with the NC and blank groups, the expression of miR-29b in the inhibitor group was down-regulated, while that of the mimics group was upregulated $(P<.05)$ (Figure 3A). It revealed that the miR-29b mimics and inhibitors were successfully transfected. The expression levels of Notch1, DII4 and Hesl mRNA in the inhibitor group were down-regulated, and those of the mimics group were upregulated $(P<.05$ versus the NC and blank groups) (Figure $3 \mathrm{~B})$. It illustrated that upregulation of miR-29b could increase the expression of Notch1, DII4 and Hesl, and activate the Notch signaling pathway significantly.

The expression of NICD1 protein: There was no difference of NICD1 expression in myocardium tissue between the sham and normal groups. Compared with the sham and normal groups, the NICD1 expression in the AMI group was significantly increased $(P<.05)$ (Figure 4A). It illustrated that myocardial infarction could increase the expression of NICD1. In H9c2 (2-1) cell line, there was no difference of NICD1 expression between the blank and NC groups $(P>.05)$. The expression of NICD1 in mimics group was increased, while that of the inhibitor group was decreased $(P<$ .05 versus the blank and NC groups) (Figure 4B). It explained that miR-29b could increase the expression of NICD1.

\section{DISCUSSION}

In this study, the rat AMI model was successfully constructed. Compared with the sham and normal groups, the relative miR-29b expression was reduced, and the expression levels of Notch1, DII4, Hesl and NICD1 were up-regulated in the AMI group. miR-29b upregulation could promote the expression levels of Notch1, DII4, Hesl and NICD1. Simultaneously, down-regulated miR-29b inhibited the expression levels of Notch1, DII4, Hesl and NICD1.

After MI, myocardial fibroblasts differentiate into myofibroblasts, which lead to excessive deposition of extracellular matrix [Melchiorbecker 2011]. Melchiorbecker et al have confirmed that miR-29b was mainly expressed in myocardial fibroblasts, and closely related to myocardial fibrosis [E 2008]. For instance, van et al promulgated that the expression of miR-29 was decreased in MI rats, and down-regulation of miR-29 could promote cardiac fibrosis by increasing the expression of collagens [E 2008]. In addition, Zhang et al found that miR-29b inhibited cardiac fibrosis via TGF- $\beta /$ Smad 3 signaling [Zhang 2014]. Moreover, miR-29 could inhibit the CDK2 and cyclin E complex, further suppressed the progression of cardiac fibrosis [Qi 2017]. And cardiac fibrosis was induced by LPS through down-regulating the expression of miR-29c and up-regulating NOX2 expression [Lew 2014]. Besides, Lin et al also indicated that miR-29b could inhibit myocardial fibrosis via mediates carvedilol [Lin 2012]. Consistent with previous studies, the relative miR-29b expression was downregulated in this study. And we also found the expression levels of Notch1, DII4, Hesl and NICD1 were upregulated in the AMI group, indicating that the Notch signaling pathway was activated in rats with AMI. Jin et al also promulgated that Notch signaling pathways were activated after myocardial infarction [Jin 2017]. Notch signaling was involved in cardiac repair and regeneration after myocardial infarction [Li 2010]. At the same time, Notch signaling could switch cardiac repair by transforming a pro-fibrotic default pathway 
to a procardiogenic one [Nistri 2017]. Furthermore, Notch signaling might inhibit the transformation from cardiac fibroblast to myofibroblast, which could prevent the progression of myocardial fibrosis [Fan 2011]. In this study, we propose a hypothesis that overexpression of miR-29b could promote the expression levels of Notch1, DII4, Hesl and NICD1, and the results in the inhibitor group were opposite. Whereby, miR-29b could inhibit myocardial fibrosis through the Notch signaling pathway. It has been found that injection of adenovirus carrying NICDl gene after myocardial infarction could improve cardiac hemodynamics [Gude 2008]. In addition, overexpression of NICD1 could improve heart repair after AMI through Notch1 signaling [Chen 2010]. And the infarct size of the infarcted rat with half deletion Notchl gene was larger than that of the control group, and the heart function was deteriorated [Li 2011]. Besides, the myocardial hypertrophy response in rats with Notchl knockout was aggravated [Croquelois 2008]. Melatonin could regulate Notch1 pathway, and then against myocardial infarction remodeling [Pei 2016]. All the above studies suggest the upregulation of the Notch signal was an adaptive response of the heart to injury and it could be a protective effect on cardiac myocytes.

In this study, we studied the effect of miR-29 on cardiac fibrosis via Notch1 pathway. But the mechanism of miR-29 regulating cardiac fibrosis is complex, and there are still many unexplored areas. In the future, more miRNAs and related pathways will be researched for cardiac fibrosis. In addition, other molecular mechanisms, such as lncRNA, will be included. Briefly speaking, miR-29b could inhibit myocardial fibrosis and cardiac hypertrophy by activating the Notch signaling pathway, which protected myocardium after myocardial infarction. It might provide a therapeutic opportunity for heart failure after myocardial infarction at the genetic level.

\section{REFERENCES}

Chen L, Anjak A, Zhou M, et al. 2010. Abstract 21055: Direct Nicd1 Overexpression Enhances Cardiac Commitment of Cpcs and Improve Heart Repair After Acute Myocardial Infarction via Notch1/gata4 Signaling. Circulation 122:A21055.

Chen X, Xiao W, Chen W, et al. 2017. MicroRNA-26a and -26b inhibit lens fibrosis and cataract by negatively regulating Jagged-1/Notch signaling pathway. Cell Death \& Differentiation 24:1431.

Croquelois A, Domenighetti AA, Nemir M, et al. 2008. Control of the adaptive response of the heart to stress via the Notch1 receptor pathway. Journal of Experimental Medicine 205:3173-85.

E vR, LB S, JE T, et al. 2008. Dysregulation of microRNAs after myocardial infarction reveals a role of miR-29 in cardiac fibrosis. Proceedings of the National Academy of Sciences of the United States of America 105:13027-32.

Fan YH, Dong H, Pan Q, et al. 2011. Notch signaling may negatively regulate neonatal rat cardiac fibroblast-myofibroblast transformation. Physiological Research 60:739-48.

Gopalakrishnan N, Sivasithamparam ND, Devaraj H. 2014. Synergistic association of Notch and NFKB signaling and role of Notch signaling in modulating epithelial to mesenchymal transition in colorectal adenocarcinoma. Biochimie 107:310-8.
Gude NA, Emmanuel G, Wu W, et al. 2008. Activation of Notchmediated protective signaling in the myocardium. Circulation Research 102:1025-35.

Jin JL, Deng ZT, Lyu RG, et al. 2017. [Expression changes of Notch and nuclear factor- $\kappa \mathrm{B}$ signaling pathways in the rat heart with myocardial infarction]. China Medical Abstracts (Internal Medicine) 45:507-12.

Lew WY, Bayna E, Dalle ME, et al. 2014. Myocardial fibrosis induced by exposure to subclinical lipopolysaccharide is associated with decreased miR-29c and enhanced NOX2 expression in mice. Plos One 9:e107556.

Li Y, Hiroi Y, Liao JK. 2010. Notch Signaling as an Important Mediator of Cardiac Repair and Regeneration After Myocardial Infarction. Trends in Cardiovascular Medicine 20:228-31.

Li Y, Hiroi Y, Ngoy S, et al. 2011. Notch1 in Bone Marrow-derived Cells Mediates Cardiac Repair Following Myocardial Infarction. Circulation 123:866.

Lin QX, Yong-Heng FU, Huang H, et al. 2012. miR-29b mediates effect of carvedilol on inhibition of acute myocardium infarction-induced myocardial fibrosis. Chinese Journal of Pathology Physiology.

Melchiorbecker A, Dai G, Ding Z, et al. 2011. Deficiency of Biglycan Causes Cardiac Fibroblasts to Differentiate into a Myofibroblast Phenotype. Journal of Biological Chemistry 286:17365-75.

Nistri S, Sassoli C, Bani D. 2017. Notch Signaling in Ischemic Damage and Fibrosis: Evidence and Clues from the Heart. Frontiers in Pharmacology 8 .

Pei H, Du J, Song X, et al. 2016. Melatonin prevents adverse myocardial infarction remodeling via Notch1/Mfn2 pathway. Free Radical Biology \& Medicine 97:408-17.

Qi H, Liu Y, Li S, et al. 2017. Activation of AMPK Attenuated Cardiac Fibrosis by Inhibiting CDK2 via p21/p27 and miR-29 Family Pathways in Rats. Molecular Therapy Nucleic Acids 8:277-90.

Smrčka M, Šána J, Fadrus P, et al. 2016. Global microRNA Expression Analysis of Sox-2 positive andNegative Glioblastoma Cells. 76:1959-.

Wang Z, Dong Y, Lan Y. 2016. Dose response of spironolactone improved ventricular remodeling after acute myocardial infarction. Journal of Cardiovascular \& Pulmonary Diseases.

Wu F, Yu B, Zhang X, et al. 2017. Cardioprotective effect of Notch signaling on the development of myocardial infarction complicated by diabetes mellitus. Experimental \& Therapeutic Medicine 14:3447.

Yanbing LI, Qiang LI, Guan X, et al. 2016. Analysis of acute myocardial infarction caused by coronary artery occlusion. Journal of Clinical Cardiology.

Yang F, Li P, Li H, et al. 2015. microRNA-29b Mediates the Antifibrotic Effect of Tanshinone IIA in Postinfarct Cardiac Remodeling. J Cardiovasc Pharmacol 65:456-64.

Zhang Y, Kanter EM, Yamada KA. 2010. Remodeling of Cardiac Fibroblasts Following Myocardial Infarction Results in Increased Gap Junction Intercellular Communication. Cardiovascular Pathology the Official Journal of the Society for Cardiovascular Pathology 19:e233.

Zhang Y, Huang X, Wei L, et al. 2014. miR-29b as a Therapeutic Agent for Angiotensin II-induced Cardiac Fibrosis by Targeting TGF- I [beta] I/ Smad3 signaling. Molecular Therapy the Journal of the American Society of Gene Therapy 22:974-85.

Zhao S, Xiao X, Sun S, et al. 2018. MicroRNA-30d/JAG1 axis modulates pulmonary fibrosis through Notch signaling pathway. Pathology Research and Practice.

Zhou X, Chen X, Cai JJ, et al. 2015. Relaxin inhibits cardiac fibrosis and endothelial-mesenchymal transition via the Notch pathway. 9:4599-611. 